\title{
Simulador de vuelo ejecutivo como medio de aprendizaje en la planeación de recursos de nuevas empresas bajo el enfoque del marcador balanceado
}

\author{
Executive flight simulator as a learning tool in new companies' resource \\ planning based on the balanced scorecard
}

\author{
Daniela Vidal Flores ${ }^{\mathrm{a}, *}$ y Rogerio Domenge Muñoz ${ }^{\mathrm{b}}$ \\ a Twentieth Century Fox Home Entertainment, Ciudad de México, México \\ b Instituto Tecnológico Autónomo de México, Ciudad de México, México
}

Recibido el 25 de febrero de 2015; aceptado el 28 de enero de 2016

Disponible en Internet el 4 de febrero de 2017

\section{Resumen}

Una deficiente planeación estratégica en las empresas de nueva creación ha generado muchas veces que las decisiones iniciales de los emprendedores no hayan sido las adecuadas y que, a la larga, las consecuencias se vean reflejadas en el fracaso de muchos nuevos negocios. El presente artículo tiene como objetivo proponer un simulador de vuelo ejecutivo que permita identificar y evaluar distintas estrategias de desarrollo de recursos de una nueva empresa manufacturera bajo las cuatro perspectivas del marcador balanceado, sensibilizando al usuario en el impacto que estas tendrían en los principales indicadores de desempeño. El simulador está diseñado utilizando el enfoque de dinámica de sistemas, para ser utilizado didácticamente en programas de maestría en administración, emprendedores o de desarrollo ejecutivo.

(C) 2017 Universidad Nacional Autónoma de México, Facultad de Contaduría y Administración. Este es un artículo Open Access bajo la licencia CC BY-NC-ND (http://creativecommons.org/licenses/by-nc-nd/4.0/).

Códigos JEL: C63; G31; L26; M13

Palabras clave: Simulador de vuelo ejecutivo; Emprendedores; Análisis de escenarios; Desarrollo de recursos; Marcador balanceado; Dinámica de sistemas

\footnotetext{
* Autor para correspondencia.

Correo electrónico: daniela.vidal@fox.com (D. Vidal Flores).

La revisión por pares es responsabilidad de la Universidad Nacional Autónoma de México.
} 


\section{Abstract}

A deficient strategic planning in new companies has produced that entrepreneurs initial decisions haven't been the appropriate and, in the long term, consequences will be reflected in the failure of many new companies. The objective of this article is to propose an executive flight simulator that will help to identify and evaluate different strategies for the development of resources of a new manufacturing company, based on the four perspectives of the balanced scorecard that will help the user to become sensitive in the impact that this will have in the performance measures. The simulator is designed under the System Dynamics view for educational purposes in MBAs, entrepreneurship or executive development programs.

(C) 2017 Universidad Nacional Autónoma de México, Facultad de Contaduría y Administración. This is an open access article under the CC BY-NC-ND license (http://creativecommons.org/licenses/by-nc-nd/4.0/).

JEL classification: $\mathrm{C} 63 ; \mathrm{G} 31 ; \mathrm{L} 26 ; \mathrm{M} 13$

Keywords: Executive flight simulator; Entrepreneurship; Scenario analysis; Resource development; Balanced scorecard; System dynamics

\section{Introducción}

Uno de los principales problemas que enfrenta el emprendedor actual es el diseño e implementación de estrategias exitosas (Domenge y Belausteguigoitia, 2009) que le permitan crear alguna ventaja competitiva relativamente sostenible, identificando la forma de invertir o asignar sus escasos recursos financieros de la manera más adecuada. Estas estrategias, por su propia naturaleza holística, deberían considerar los principales recursos estratégicos de la empresa de todas sus áreas funcionales (humanos, tecnológicos, operativos y de mercadotecnia) que pudiera necesitar, así como la forma y la velocidad para hacerse de ellos, desarrollándolos o acumulándolos de manera complementaria (Sterman, 2007) y equilibrada (Warren, 2005).

Otro problema que se le presenta al emprendedor es la evaluación de los impactos esperados derivados de sus posibles estrategias, visualizados de una forma comprensible y sintética, reflejando los valores de diversos indicadores de desempeño considerados para evaluar o comparar dichas estrategias respecto a los objetivos esperados, como pudiera ser el valor presente neto (VPN) de cada una de ellas.

La identificación de las relaciones que existen entre los recursos estratégicos disponibles para el emprendedor, su estrategia equilibrada de desarrollo y los indicadores de desempeño que proporcionen sus posibles impactos conforman las bases de un simulador de vuelo ejecutivo (SVE) que puede ser de utilidad para el emprendedor en el momento de evaluar y sensibilizarse en el diseño y la determinación de la estrategia más conveniente para su nueva empresa.

En los últimos años ha aumentado la tendencia a hacer planes de negocios como parte de la planeación inicial de un nuevo negocio, debido, entre otras razones, a que es un requisito para solicitar un crédito o invitar a socios capitalistas o estratégicos, terminando, en muchas ocasiones, en un documento estático hecho de la agregación de datos, lo cual no permite a los emprendedores entender la estructura del sistema dinámico en el cual operará la empresa (Bianchi, Winch y Grey, 1998).

La planeación de negocios en un contexto de aprendizaje permite a los emprendedores prever los escenarios futuros de crecimiento del negocio y, por consiguiente, entender el tiempo adecuado para empezar a construir recursos estratégicos relevantes que le permitirán a la empresa moverse hacia escenarios deseados subsecuentes. El plan de negocios tradicional puede ser considerado 
como herramienta de aprendizaje al complementarlo con un SVE que permita a los emprendedores, entre otras cosas, detectar los límites de crecimiento del negocio relacionados con la escasez de recursos (Bianchi et al., 1998, Bianchi, 2002). El enfoque al aprendizaje en el desarrollo de los planes de negocio permite el cuestionamiento de los modelos mentales (Senge, 1994) del emprendedor a través de la generación de nuevas visiones acerca de cómo el negocio se comportaría en el futuro, como consecuencia de las decisiones actuales (Bianchi, 2002) mejor informadas.

El aprendizaje por experiencia es un proceso de retroalimentación que muchas veces es tardado y costoso. Se toman decisiones que afectan los resultados reales, se recaba información de la retroalimentación y con ella se revisa lo que se está entendiendo del proceso y de las decisiones que se están tomando para alcanzar los objetivos (Sterman, 2000). Los SVE son una forma práctica de analizar y tomar decisiones más informadas sobre el futuro del negocio. Los altos niveles competitivos y las exigencias del mercado obligan a los emprendedores a estar más preparados y a tener mayores conocimientos para poder hacer frente a los retos de emprender. Debido a que una nueva empresa debe estar en constante aprendizaje y crecimiento, el SVE proporciona un medio para sensibilizar al emprendedor mediante una retroalimentación inmediata del impacto de sus estrategias, permitiendo analizar los efectos individuales y conjuntos que podrían tener las decisiones tomadas sobre los recursos con los que cuenta la empresa. Asimismo, los sistemas interactivos, entre los cuales se encuentran los simuladores, ayudan a mejorar los procesos de planeación y control y juegan el rol de un campo de práctica para los emprendedores antes de llevar a cabo una acción en el mundo real. Los simuladores permiten a los emprendedores mejorar el entendimiento del sistema como un todo, mejorar la comunicación entre los involucrados, explotar la experiencia del emprendedor y su percepción del negocio, identificar las políticas que afectan el desempeño de corto y largo plazo y mejorar la interacción continua (Bianchi et al., 1998, Bianchi, Bivona y Landriscina, 2000).

El presente artículo propone un SVE que incluye variables decisionales respecto al desarrollo de diversos recursos bajo las cuatro perspectivas (Kaplan y Norton, 1992) del marcador balanceado (MB). Dicho SVE pretende que estudiantes de alguna materia de estrategia emprendedora, ya sean alumnos a nivel licenciatura, maestría, ejecutivos o emprendedores, puedan aplicar sus conocimientos y experiencia en el diseño de una estrategia adecuada de crecimiento de una nueva empresa manufacturera, considerando las limitantes que puedan representar los propios recursos entre sí mismos cuando no se desarrollan equilibradamente, considerando los cuellos de botella que pudieran surgir al desarrollar más algunos de ellos respecto a los otros.

Los objetivos didácticos del SVE propuesto en este documento son que el estudiante:

- Identifique los principales indicadores de desempeño a utilizar en la evaluación de los impactos que tendrían sus decisiones estratégicas de desarrollo de recursos.

- Identifique los recursos con los que cuenta bajo las cuatro perspectivas del MB.

- Explique las relaciones entre los recursos y los indicadores de desempeño.

- Proponga y evalúe estrategias de crecimiento que desarrollen equilibradamente sus recursos, identificando su impacto en los indicadores de desempeño considerados en el modelo.

\section{Revisión de la literatura}

El proceso que generalmente siguen los emprendedores potenciales es arduo, complicado y en no pocas ocasiones desilusionante. Los emprendedores, individuos que creen poseer las capacidades y habilidades necesarias para iniciar un negocio, aquellos que ven oportunidades 
para emprender y que no serán disuadidos de iniciar un negocio por miedo al fracaso (GEM, 2012), pasan por una serie de etapas iterativas en su proyecto emprendedor, que incluyen desde una visión inicial más o menos clara de la nueva empresa que desean crear y la forma o estrategia de cómo lo van a lograr, hasta las diferentes actividades o iniciativas prácticas que van a realizar con el objetivo de lograr una exitosa implementación de la estrategia.

Emprender es un proceso dinámico de decisiones. Con base en información disponible, conocimiento, talento y experiencia, los emprendedores deciden explorar una oportunidad que anteriormente identificaron. El proceso de creación de valor de una empresa comienza en la cultura, el liderazgo y la mentalidad del emprendedor, la aplicación de su creatividad y el desarrollo de ideas innovadoras, buscando obtener alguna ventaja competitiva a partir de la identificación y administración de ciertos recursos estratégicos que a mediano o largo plazo llevará a la creación de riqueza (Pina, 2007).

La planeación estratégica le permite al emprendedor explorar posibles escenarios futuros del crecimiento y desarrollo del negocio, entendiendo cuándo sería el tiempo adecuado para construir sus recursos (por ejemplo, personal capacitado, estructura organizacional, sistemas de manufactura, reputación y valor de la marca, desarrollo de cartera de clientes, etc.) que le permitirán a la empresa avanzar hacia un sano desarrollo. Dicho crecimiento de la empresa también se puede analizar externamente, considerando las tendencias de su entorno y definiendo el valor de lo que ofrece la empresa, de acuerdo a las características de la demanda de su mercado actual y potencial. Entre mejor comprenda el emprendedor la estructura y funcionamiento de su sistema (su empresa y su entorno) en el que opera, mejores posibilidades tendrá en lograr éxito. Por lo tanto, las empresas necesitan estar en continuo aprendizaje, como prerrequisito de su sano desarrollo (Senge, 1994).

\section{El emprendedor y el manejo de recursos}

El análisis estratégico de los recursos se puede basar en la teoría basada en recursos (Resource Based View [RBV]; Rugman y Verbeke, 2002), la cual es una teoría de la organización enfocada en la identificación y desarrollo de los recursos y capacidades controladas por la organización como fuentes de ventaja competitiva. Los recursos son todos los activos tangibles e intangibles que la empresa controla total o parcialmente y que pueden ser utilizados para concebir e implementar su estrategia (Barney y Hesterly, 2012; Warren, 2008; Carroll, 1993). Las capacidades son un subconjunto de los recursos de la empresa y se definen como las ventajas tangibles e intangibles que permiten a la empresa obtener toda la ventaja de sus otros recursos.

Existen varias clasificaciones de los recursos en la empresa u organización: Barney y Hesterly (2012) los clasifican en cuatro categorías: recursos financieros, incluyendo todo el dinero que la empresa utiliza para desarrollar e implementar sus estrategias; recursos físicos, siendo todas las tecnologías físicas utilizadas en la empresa, como la planta y equipo, la localización geográfica y el acceso a materias primas; recursos humanos, incluyendo la experiencia, la capacitación y las relaciones de todos los trabajadores de la empresa, y recursos organizacionales, constituidos por la estructura de la organización, el proceso de planeación, los sistemas de coordinación, cultura, incentivos y reputación.

Otro enfoque, más orientado al emprendedor, es el de Dollinger (2008), que define seis tipos de recursos, conocidos como el esquema PROFIT Factors: 
- Recursos físicos: aquellos activos tangibles utilizados para la producción y la administración de la empresa, incluyendo maquinaria y equipo, localización geográfica y materia prima.

- Recursos de reputación, constituidos por las percepciones que la gente en el ambiente laboral tiene de la empresa; incluyen también la reputación a nivel de producto como lealtad de marca o a nivel corporativo como imagen global.

- Recursos organizacionales, considerando a toda la estructura de la empresa, sus procesos y sistemas.

- Recursos financieros: todos los activos monetarios; representan la capacidad de pedir dinero prestado, la habilidad de crear nuevo capital y la cantidad de dinero generada por las operaciones internas. La administración de los recursos financieros (la organización, los procesos y rutinas) que ayuda a usar los recursos de una manera más eficiente es la que genera una ventaja competitiva sostenible, ya que el dinero es un recurso estático e inherente, pero la habilidad de manejarlo es dinámica, compleja y creativa.

- Recursos intelectuales y humanos, incluyendo el conocimiento, la capacitación y la experiencia del emprendedor y de su equipo. En este tipo de recursos se incluye la creatividad, visión e inteligencia de los miembros de la organización, así como las habilidades sociales y personalidad del emprendedor.

- Recursos tecnológicos y operativos de los procesos: conocimientos generados a partir de la experiencia y de la investigación y desarrollo y generalmente protegidos con patentes, licencias y derechos de autor.

De acuerdo con Barney y Hesterly, 2012 y Warren (2008), la teoría basada en recursos propone que una ventaja competitiva sostenible es creada cuando la empresa posee y emplea recursos y capacidades que siguen el esquema VRIO, es decir, que estos sean valiosos: ayuda a la organización a implementar su estrategia, permitiéndole aprovechar o explotar una oportunidad o neutralizar una amenaza del entorno; raros: no está disponible para la competencia o muy pocas empresas controlan el recurso; inimitables: el recurso es difícil de duplicar o resulta muy costoso, y aprovechados por la organización: la empresa está organizada (políticas y procesos) para explotar totalmente el potencial competitivo de sus recursos y capacidades. El recurso humano más importante y valioso que un nuevo negocio tiene es el emprendedor, ya que cada uno tiene sus características especiales, historias, personalidad, experiencias y relaciones sociales únicas (Dollinger, 2008; Timmons, 2009).

El diseño de la estrategia, bajo el enfoque basado en recursos, contempla las decisiones que afectan las tasas de ganancia y pérdida de cada recurso en el tiempo, explicando la cantidad de cada recurso en cualquier momento en el tiempo, concepto de stock-and-flow de Akkermans y Oorschot, 2005. El desempeño del sistema depende de los niveles de los recursos, y estos dependen, a su vez, de las entradas y salidas de cada recurso. Lo que guía el flujo de los recursos y su desarrollo o evolución (o posible involución) son principalmente las decisiones de los directivos, los factores externos, los impactos o contribuciones de cada recurso en los indicadores de desempeño (sensibilidad) y los niveles existentes relativos entre ellos (Warren, 2008).

Los recursos se construyen por el flujo de entradas al nivel de recurso considerado (por ejemplo, nuevos clientes, contrataciones, nuevos productos o servicios, tecnología, capacidad de producción) y por las pérdidas sufridas por errores, acciones de terceros, decisiones tomadas (clientes que se van con la competencia, empleados que renuncian o son despedidos, pérdida de conciencia de marca, productos discontinuados, depreciación). El emprendedor determina la estrategia basándose en el concepto de asset-stock accumulation, siendo la característica de los recursos la de aumentar y disminuir con el transcurso del tiempo. Esta característica es crítica para el 
desempeño estratégico y es también conocida como integración en la teoría de control (Ogatta, 2009). La cantidad actual de un recurso es causa de la cantidad inicial del siguiente periodo más lo que se agrega y disminuye en el mismo período (Warren, 2005).

El crecimiento y decrecimiento de un recurso dependerá también del nivel de otros recursos (Warren, 2008), los cuales pueden limitarse unos a otros causando ineficiencias, cuellos de botella y disminución de productividad. Por ejemplo, en una empresa consultora, el número de consultores senior dependerá del número de consultores junior y de su promoción. Otro ejemplo es el nivel de producción en una empresa manufacturera, que depende tanto de la capacidad física instalada como de la cantidad de los empleados. En este último ejemplo el nivel relativo de cada recurso es importante, ya que si se encuentran en desequilibrio, habrá un desperdicio del recurso con un mayor nivel. Esta situación también se puede analizar al desarrollar los recursos, ya que si en el momento $t$ se encuentran en equilibrio, al desarrollarlos a futuro, en el tiempo $t+n$, posiblemente uno se desarrolle más rápidamente que el otro (uno tenga más retraso o delay en su desarrollo) llegando a una situación o estado de desequilibrio y, por lo tanto, menos eficiente o productivo.

Otros ejemplos de desarrollo interdependiente de recursos son: desarrollo y lanzamiento de nuevos productos con su múltiples etapas desde la concepción de la idea hasta su lanzamiento; la capacitación de personal con etapas o niveles de capacitación; en general los recursos con una cierta vida útil, que dependen de su propio nivel, del tiempo de vida, de su velocidad de regeneración y de los niveles de otros recursos que afectan los flujos de entrada y salida (ciclo de vida de producto, tecnología, empresa, etc.); niveles de adopción de un cierto producto por tipo de cliente (Rogers, 1962); cadena de distribución con sus niveles relativos entre etapas desde la producción hasta el cliente y el efecto látigo (juego de la cerveza, Sterman, 2000); nivel de vida de una población que depende de recursos como nivel educativo, seguridad, alimentación, etc. Por lo tanto, los sistemas dinámicos hacen explícita la naturaleza de «complementariedad» entre los recursos, tanto tangibles como intangibles. La complementariedad se refiere a la manera en que los recursos se crean e interaccionan entre sí, condicionando el desempeño global del sistema a sus niveles relativos (Warren, 2008; Sterman, 2007).

Los sistemas dinámicos no limitan su análisis a los recursos que le «pertenecen»o «controla» la empresa. Para afectar el desempeño, una empresa requiere únicamente tener acceso limitado a los recursos que necesita. Una consecuencia de ello es que los clientes se vuelven parte del sistema, aunque no le pertenecen ni los controla directa o totalmente la empresa.

\section{El marcador balanceado}

La complejidad de una organización requiere que los directivos sean capaces de diseñar una estrategia y ver el desempeño en diversas áreas de manera simultánea. El balanced-scorecard o marcador balanceado es una herramienta que proporciona un marco de referencia para diseñar la estrategia y traducirla en acción. Incluye medidas financieras que muestran los resultados de las acciones tomadas y las complementa con medidas operacionales considerando al cliente, a los procesos internos, innovación y recursos humanos (Kaplan y Norton, 1992). Una de las características del $\mathrm{MB}$ es que sirve de puente entre distintas áreas, tanto financieras como no financieras (Akkermans y Oorschot, 2005).

El MB permite observar a la empresa desde cuatro perspectivas: la perspectiva de aprendizaje y crecimiento, reflejada en la habilidad de la empresa para aprender, innovar y mejorar los productos y procesos ya existentes e introducir nuevos productos; la perspectiva de procesos internos $\mathrm{u}$ operaciones internas que se llevan a cabo para elaborar los productos $\mathrm{u}$ ofrecer los servicios para satisfacer las necesidades del consumidor; la perspectiva del cliente, orientada a crear valor 
y diferenciación, y la perspectiva financiera, que proporciona indicadores del desempeño de la estrategia, su implementación y su ejecución desde el punto de vista monetario, crecimiento, rendimiento y riesgo.

El MB facilita la determinación de un panorama a futuro, definir una visión estructurada y establecer un camino para llegar a ella (un mapa estratégico), desarrollando los recursos existentes o clasificados de acuerdo a las cuatro perspectivas, así como diseñar un tablero con los objetivos y acciones (implementación) cuantificables en el corto y mediano plazo.

El MB puede ser capturado en un modelo de dinámica de sistemas (Bianchi et al., 2000; Warren, 2008) que brinde una representación comprensible y cuantificable sobre el proceso de creación de valor en el tiempo para un negocio, identificando los recursos necesarios desde sus cuatro perspectivas, identificando el tiempo y la velocidad de su creación o desarrollo, así como las acciones o decisiones necesarias y sus correspondientes impactos medidos mediante los indicadores de desarrollo, que se definen también bajo el esquema de sus cuatro perspectivas. El uso de MB respalda la importancia del entendimiento de las relaciones causa-efecto, recursosmedidas de desempeño, entre los componentes de la estrategia de le empresa, los cuales tienen un impacto en las medidas de desempeño financieras (Capelo, 2009).

El MB puede ayudar a una organización de varias formas, promoviendo el crecimiento con enfoque, claridad de objetivos y metas, alineación organizacional, monitoreo del desempeño evaluando la efectividad en el logro de objetivos e identificando responsabilidades y responsables en los resultados esperados (Gumbus y Lussier, 2006). Los tomadores de decisiones estratégicas pueden utilizar herramientas como el MB que les ayuden a definir la estrategia identificando sus recursos desde las cuatro perspectivas y que los mantenga informados de los avances en su implementación (Ritchie-Dunham, 2001).

\section{El entorno de aprendizaje}

Los entornos de aprendizaje interactivo, o interactive learning environments, son medios que permiten el logro de objetivos de aprendizaje mediante la interacción activa del participante y el sistema focal (Domenge, 2009; Domenge y Vidal, 2015). En particular, los SVE son un tipo de entornos de aprendizaje interactivo diseñados para ser utilizados en la computadora, bajo la estructura de un juego interactivo, basados en simuladores virtuales de sistemas reales y que utilizan una interfaz amigable entre el sistema y el tomador de decisiones (Kopainsky, Alessi, Pedercini y Davidsen, 2009; Maier y Gröbler, 2000). Estos sistemas ofrecen un ambiente de aprendizaje activo para lograr un conjunto de tareas y objetivos, brindando el soporte necesario para ayudar a aprender y aplicar uno o más conceptos específicos (Aleven, Stahl, Schworm, Fischer y Wallace, 2003; Choi y Hannafin, 1995).

Un SVE es cualquier intento de representar un ambiente o un sistema real o imaginario en un modelo computacional de simulación. Un simulador tiene dos propósitos principales: científico y educativo (Davidsen, 2000). El diseño y la utilización de un simulador implican una o más razones por las cuales no se experimenta directamente con el sistema real, tales como costo, tiempo, riesgo o falta de acceso a los recursos. Los simuladores científicos brindan un panorama de un sistema real que permite a los realizadores establecer y mejorar la teoría existente para un mejor desarrollo del mismo sistema. Los simuladores educativos son diseñados como medio de aprendizaje virtual acerca de la estructura y del funcionamiento de un sistema mediante la evaluación de los resultados obtenidos a partir de acciones o decisiones tomadas, a través de la retroalimentación generada por el simulador en tiempo real, tiempo acelerado o tiempo más lento (Rieber, 1996). 


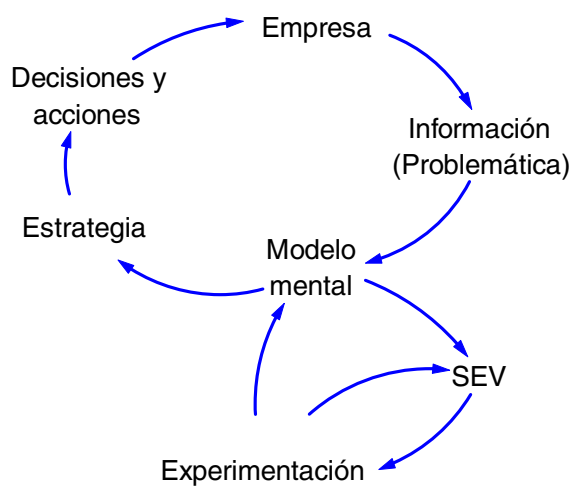

Figura 1. Papel del SVE en la estrategia.

Los SVE son medios para proveer aprendizaje en la educación empresarial, representando «campos de práctica» o experimentación para los tomadores de decisiones en la empresa (fig. 1) y creando un ciclo de aprendizaje efectivo, ya que cierran la brecha entre el diseño e implementación de estrategias y sus correspondientes efectos. Los SVE permiten modificar iterativamente el modelo mental (Senge, 1994) del estratega, el cual le permite comprender conceptualmente la estructura y el funcionamiento del sistema (empresa y entorno) en el que se encuentra (parte inferior de la fig. 1), al experimentar y evaluar los impactos de diversas estrategias antes de decidir por la más conveniente.

Al utilizar un SVE se presentan dos tipos de retroalimentación y aprendizaje: el single-loop y el double-loop (Bianchi, 2002). El primero es el tipo de aprendizaje básico, en el cual la retroalimentación de los impactos de las decisiones se basa en la experiencia con el sistema real y es interpretada por los modelos mentales existentes. El aprendizaje opera en el contexto de las reglas de decisión existentes, estrategias, cultura e instituciones (rizo superior de la fig. 1). En el aprendizaje tipo double-loop la retroalimentación del mundo real puede también estimular cambios en los modelos mentales, pero dicho aprendizaje involucra nuevos conceptos de una situación y lleva a nuevos objetivos y reglas de decisión, y no únicamente a nuevas decisiones (Sterman, 2000; Lane, 1995). El aprendizaje tipo double-loop permite a los tomadores de decisiones evaluar las consistencias de sus modelos mentales, ya que uno de los riesgos más importantes es el tomar decisiones sin haber cuestionado la consistencia del modelo mental (Bianchi et al., 1998; Bianchi, 2002; Bianchi et al., 2000).

Los SVE generan un ambiente dinámico que presenta una o más situaciones que requieren decisiones, creando ciclos y nuevas situaciones con nuevos problemas por resolver y decisiones a tomar (Wawer, 2010). Los SVE son un método efectivo de aprendizaje sobre diversos aspectos empresariales y son considerados como uno de los más interesantes métodos de aprendizaje, ya que llevan al usuario a involucrarse y comprometerse en sus decisiones, conforme va avanzando en el uso interactivo del simulador, monitoreando resultados de inmediato, acelerando el proceso de aprendizaje y adquiriendo experiencia rápidamente, experimentando nuevos comportamientos y desarrollando ciertas habilidades y competencias relacionadas con el proceso de toma de decisiones (Saad, 2013; Lane, 1995). El simulador facilita el proceso de aprendizaje, ya que permite revisar y evaluar constante, inmediata e iterativamente diversas estrategias propuestas por el participante, tomando decisiones conforme a la nueva información generada por el propio simulador en una serie de escenarios. 
El SVE proporciona la posibilidad de conectar el conocimiento de las diferentes áreas involucradas en un negocio de forma holística y sistémica; produce una fuerte motivación para tener un aprendizaje activo o aprendizaje experiencial (análogo a la espiral de aprendizaje de Kolb, 2014: experiencia concreta, observación y reflexión de la experiencia, formación de conceptos abstractos o modelos mentales basados en la reflexión, prueba o validación de los nuevos conceptos, y repetición); combina el pensamiento estratégico y analítico y permite desarrollar la habilidad para trabajar en equipo y mejorar la comunicación interpersonal y el trabajo bajo presión. El proceso de aprendizaje por simulación lleva a mejorar los modelos mentales de los participantes y les ayuda a alcanzar una visión compartida de la realidad (Bianchi et al., 1998).

Por otro lado, los SVE tienen varias desventajas, como señala Wawer (2010): el simulador es un modelo de la realidad, es decir, está creado con ciertas simplificaciones surgidas a partir de una abstracción selectiva del sistema real; las decisiones se toman sin responsabilidad alguna, por lo que los resultados reflejados en el simulador no influyen en la situación de la realidad, lo cual puede influir en el comportamiento de los jugadores, difiriendo del que se pudiera tener en la realidad.

Dentro de la literatura relacionada con SVE existen diversos desarrollos que han servido para planear y evaluar estrategias respecto a algún tipo de empresa en particular, empresas en general (MIT Business Strategy Games; Blue Ocean Strategy Simulation [BOSS]), así como en determinadas áreas operativas como mercadotecnia (Markstrat), operaciones (Sterman, 2000) y recursos humanos (Warren, 1997). En el anexo 1 se muestra una tabla con diversos simuladores que se han hecho como herramienta de aprendizaje para tomadores de decisiones y para emprendedores. En esta tabla se muestra una síntesis del objetivo planteado para cada simulador, los módulos que intervienen, las variables decisionales que se tomaron en cuenta para el desarrollo de los mismos y las medidas de desempeño del modelo. Algunos de estos SVE están diseñados para simular áreas funcionales específicas como finanzas, recursos humanos o ventas, o también una combinación de estas, considerando las ventas o diversos elementos de los estados financieros como medidas de desempeño finales. Estos SVE no toman en cuenta una clasificación global de los recursos como ofrece el MB, ni destacan explícitamente el desarrollo equilibrado de recursos conforme sus relaciones causa-efecto y sensibilidad con las medidas de desempeño.

Los simuladores pueden ser utilizados como parte del proceso de planeación estratégica y de aprendizaje de una empresa y ayudan a los estrategas a distinguir la estructura de retroalimentación del sistema (empresa) y cómo modificar dicho sistema existente para afectar su comportamiento, buscando lograr los objetivos previamente planteados. La planeación puede ser concebida como un medio de aprendizaje y el SVE como vehículo que da soporte y un mejor entendimiento del sistema de negocio en cuestión (Bianchi et al., 1998) para la toma de decisiones más informada. Los SVE contribuyen a desarrollar en una empresa el concepto de la «organización que aprende» o learning organization, que desde principios de los años noventa (Senge, 1994) se ha ido reconociendo como un elemento importante en la estrategia, evolución, desarrollo, resiliencia y cambio organizacional.

\section{El simulador de vuelo ejecutivo propuesto}

De acuerdo con el planteamiento realizado en la introducción del presente documento, el SVE que se propone tiene cuatro objetivos didácticos orientados a estudiantes de programas para emprendedores en materias de planeación estratégica, es decir, que logren: 1) identificar los indicadores de desempeño a utilizar; 2) identificar recursos con los que cuenta bajo la perspectiva de MB; 3) explicar las relaciones entre sus recursos y las medidas de desempeño, y 4) proponer 


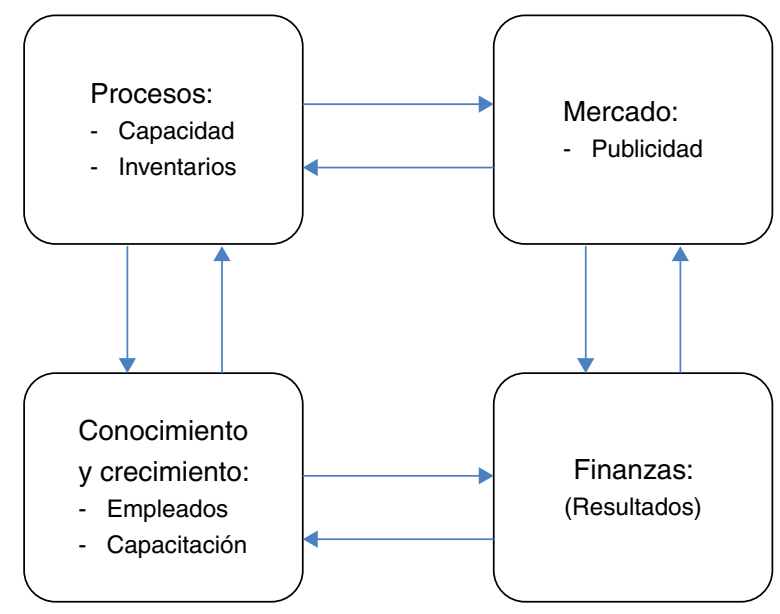

Figura 2. Estructura del SVE.

y evaluar estrategias de crecimiento que desarrollen equilibradamente sus recursos, identificando su impacto en las medidas de desempeño consideradas en el modelo.

El primer y segundo objetivos -identificación de los indicadores de desempeño e identificación de recursos con los que cuenta la empresa bajo la perspectiva de MB - se ilustran en la figura 2, que presenta los cuatro bloques que conforman la estructura del SVE. Los bloques corresponden a cada una de las cuatro perspectivas del MB. En cada bloque se indican las variables decisionales que considera el SVE orientados a desarrollar los recursos incluidos en el SVE. Los indicadores de desempeño son fundamentalmente los resultados financieros (VPN y utilidades). Los recursos a desarrollar son empleados, capacitación en productividad, capacidad de producción, inventarios y publicidad. Los niveles de los recursos también pueden ser utilizados como medidas de desempeño, ya que indican el desempeño de cada uno de ellos en el tiempo.

El tercer objetivo, las relaciones entre los recursos y las medidas de desempeño, se presentan en el diagrama causal de la figura 3 . Se incluyen las variables decisionales, los recursos considerados y la principal medida de desempeño, es decir, el VPN de los flujos generados por período de tiempo.

La estructura del modelo que constituye el SVE relaciona causalmente los recursos con los que cuenta la empresa, las variables decisionales para cada una de las cuatro perspectivas del MB y el indicador de desempeño. La producción Q está acotada por la capacidad de producción de los empleados $\mathrm{Q}_{\mathrm{E}}$ y por la capacidad de operación Qo (ecuación 1). La capacidad de los empleados $\mathrm{Q}_{\mathrm{E}}$ depende de la cantidad de empleados $\mathrm{E}$ y la productividad p por empleado (ecuaciones 2 y 3 ), la cual es una función $\mathrm{f}_{1}$ de la inversión $\mathrm{P}$ dedicada a este rubro (fig. 4A).

La capacidad de operación se ve limitada por la capacidad de operación instalada $\mathrm{K}_{\mathrm{O}}$ y el inventario deseado $\mathrm{I}_{\mathrm{D}}$ (ecuación 4).

$$
\begin{aligned}
& \mathrm{Q}=\operatorname{MIN}\left(\mathrm{Q}_{\mathrm{E}}, \mathrm{Q}_{\mathrm{O}}\right) \\
& \mathrm{Q}_{\mathrm{E}}=\mathrm{E} * \mathrm{p} \\
& \mathrm{p}=\mathrm{f}_{1}(\mathrm{P}) \\
& \mathrm{Q}_{\mathrm{O}}=\operatorname{MIN}\left(\mathrm{K}_{\mathrm{O}}, \mathrm{I}_{\mathrm{D}}\right)
\end{aligned}
$$




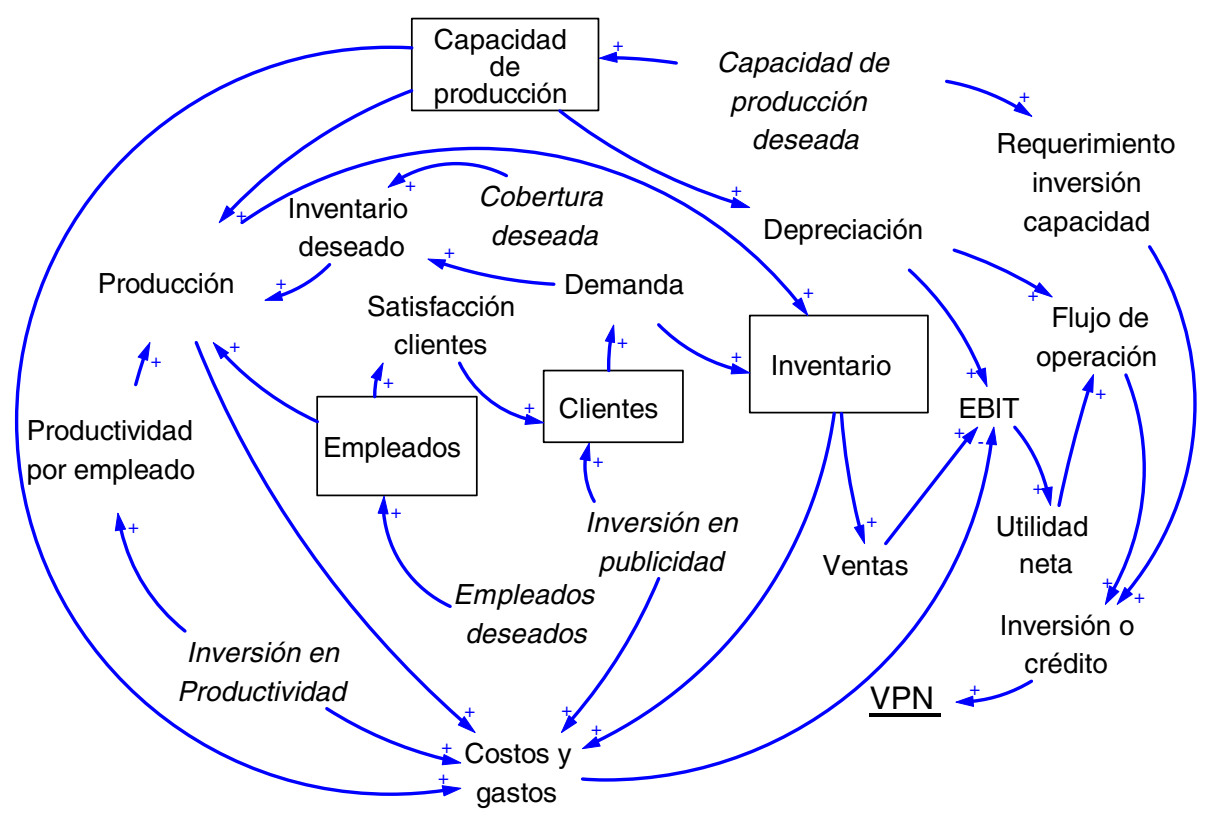

Figura 3. Diagrama causal del SVE.

El inventario deseado $\mathrm{I}_{\mathrm{D}}$ es una función de la demanda $\mathrm{D}$ y de la cobertura de inventario faltante $\mathrm{C}_{\mathrm{F}}$, que a su vez depende de la cobertura deseada $\mathrm{C}_{\mathrm{D}}$ menos la actual $\mathrm{C}$. La cobertura deseada de inventarios $C_{D}$ se logra en el tiempo $\mathrm{T}_{\mathrm{C}}$ (ecuaciones 5, 6 y 7).

$$
\begin{aligned}
& \mathrm{I}_{\mathrm{D}}=\mathrm{D} * \mathrm{C}_{\mathrm{F}} \\
& \mathrm{C}_{\mathrm{F}}=\operatorname{MAX}\left(0, \mathrm{C}_{\mathrm{D}}-\mathrm{C}\right) \\
& \mathrm{C}=\mathrm{I} / \mathrm{D}
\end{aligned}
$$

El inventario se conforma por las unidades que se producen Q menos las unidades que se venden $\mathrm{V}$, que a su vez dependen de la demanda $\mathrm{D}$, de las existencias y del tiempo que se tiene para surtir los pedidos $\mathrm{T}_{\mathrm{S}}$ (ecuación 8).

$$
\mathrm{V}=\operatorname{MIN}\left(\mathrm{D}, \mathrm{I} / / \mathrm{T}_{\mathrm{S}}\right)
$$

La capacidad de producción faltante $\mathrm{K}_{\mathrm{F}}$ depende de la capacidad deseada, de la capacidad de operación instalada y del tiempo que tarda la construcción de capacidad $\mathrm{T}_{\mathrm{K}}$ (ecuación 9).

$$
\mathrm{K}_{\mathrm{F}}=\left(\mathrm{K}_{\mathrm{D}}-\mathrm{K}_{\mathrm{O}}\right) / \mathrm{T}_{\mathrm{K}}
$$

Los empleados faltantes $\mathrm{E}_{\mathrm{F}}$ dependen de la cantidad de empleados deseados $\mathrm{E}_{\mathrm{D}}$, los empleados actuales $\mathrm{E}$ y el tiempo que tarda su contratación y capacitación $\mathrm{T}_{\mathrm{E}}$ (ecuación 10).

$$
\mathrm{E}_{\mathrm{F}}=\left(\mathrm{E}_{\mathrm{D}}-\mathrm{E}\right) / \mathrm{T}_{\mathrm{E}}
$$

El modelo propuesto considera una demanda exógena esperada D. El administrador del SVE puede programar diversos escenarios manipulando la variable de tendencia $(\mathrm{m})$ del entorno para estimar la demanda tendencial, siguiendo una función lineal respecto a la cantidad de clientes 


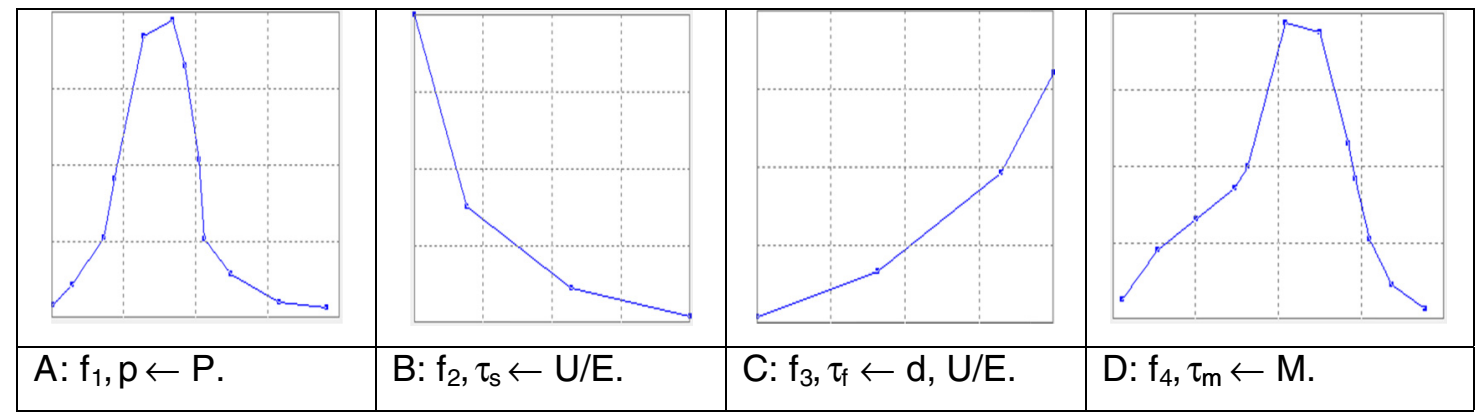

Figura 4. Relaciones entre diversas variables.

A: $f_{1}, p \leftarrow P ; B: f_{2}, \tau_{s} \leftarrow$ U/E; $C: f_{3}, \tau_{f} \leftarrow$ d, U/E; D: $f_{4}, \tau_{m} \leftarrow$ M. 
netos $\mathrm{U}_{\mathrm{N}}$ (ecuación 11). Es posible ganar o perder clientes mediante incrementos o decrementos en tres rubros (ecuación 12): en la tasa de satisfacción del cliente $\tau_{\mathrm{s}}$ estimado con una variable proxi, cantidad de clientes entre empleados (ecuación 13), por la tasa de deserción $\tau_{\mathrm{f}}$ (ecuación 14) y por la tasa de esfuerzo en mercadotecnia $\tau_{\mathrm{m}}$ debido a la inversión $\mathrm{M}$ en este rubro (ecuación $15)$.

$$
\begin{aligned}
& \mathrm{D}=\mathrm{m} * \mathrm{U}_{\mathrm{N}} \\
& \mathrm{U}_{\mathrm{N}}=\mathrm{U} *\left(\tau_{\mathrm{s}}-\tau_{\mathrm{f}}+\tau_{\mathrm{m}}\right) \\
& \tau_{\mathrm{s}}=\mathrm{f}_{2}(\mathrm{U} / \mathrm{E}) \\
& \tau_{\mathrm{f}}=\mathrm{f}_{3}(\mathrm{~d}, \mathrm{U} / \mathrm{E}) \\
& \tau_{\mathrm{m}}=\mathrm{f}_{4}(\mathrm{M})
\end{aligned}
$$

Se definió el VPN como la medida final de desempeño que evalúa cada estrategia, considerando los flujos netos $\mathrm{F}$ generados por período (incluyendo las ventas, costos fijos y variables, gastos, depreciación, impuestos e inversiones), la amortización A de la deuda por período que pudo haber surgido de la estrategia propuesta y la tasa de descuento $\alpha$ (ecuación 16).

$$
\mathrm{VPN}=(\mathrm{F}-\mathrm{A}) /\left[(1+\alpha)^{\mathrm{T}} \mathrm{Time}\right]
$$

Se diseñó el SVE bajo una perspectiva de evaluación de estrategias de crecimiento equilibrado de recursos de una empresa manufacturera de un solo producto, lo cual constituye el cuarto objetivo didáctico. Las variables decisionales (en cursiva en la fig. 3) que contiene el modelo son: la inversión en publicidad (perspectiva de mercado), la cual afecta a los nuevos clientes a través de su tasa de captación; la capacidad de producción deseada (perspectiva de procesos); la cobertura deseada de inventario sobre demanda (perspectiva de procesos), que representa el período de tiempo por el cual se desea estar cubierto para cumplir con la demanda para evitar el faltante de producto; inversión en productividad (perspectivas de aprendizaje), la cual afecta directamente a la productividad anual de los empleados y a los empleados deseados, los cuales son la fuerza laboral que se desea tener para realizar la producción.

Se pretende que cada participante proponga una estrategia base o de referencia y que posteriormente experimente o simule diversas estrategias con la finalidad de ir conformando un modelo mental que le permita relacionar sus decisiones de desarrollo de recursos con el indicador de desempeño. El objetivo no es solamente lograr un mayor VPN, sino que el participante se sensibilice sobre los impactos relativos de sus decisiones (análisis de sensibilidad del desarrollo relativo de los recursos) a medida que experimenta con el SVE, modificando su modelo mental acerca de la estructura y funcionamiento del modelo y teniendo presente las cuatro perspectivas del MB. El modelo se construyó y simuló en el paquete computacional Vensim (Ventana Systems) basado en el enfoque de dinámica de sistemas (Sterman, 2000).

La inversión $\mathrm{P}$ en productividad tiene un comportamiento creciente hasta llegar a un punto máximo en el cual inicia un decrecimiento, ya que se considera que existiría saturación (fig. 4A). Las funciones para $\tau_{\mathrm{s}}, \tau_{\mathrm{d}}, \tau_{\mathrm{m}}$ están definidas por medio de la herramienta LOOKUP del Vensim. $\mathrm{Al}$ incrementarse el nivel de satisfacción se incrementan los clientes y se decrementa la deserción (fig. 4B,C). Al invertir en los esfuerzos de mercadotecnia $\mathrm{M}$ se incrementan los nuevos clientes hasta que se saturan y los efectos se vuelven decrecientes (fig. 4D). 


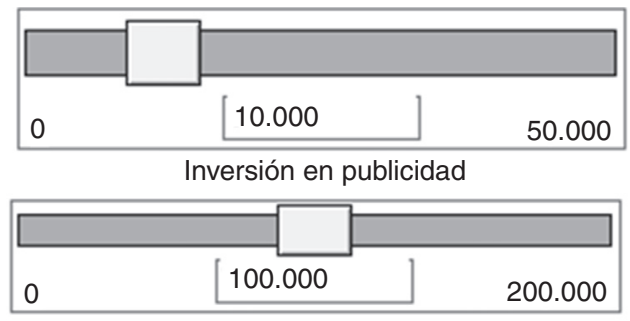

Capacidad deseada de producción en unidades

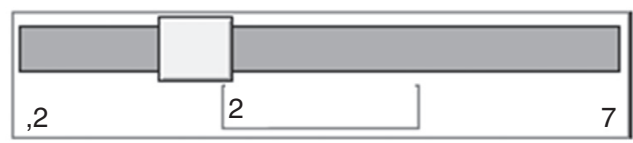

Cobertura deseada de inventario sobre demanda

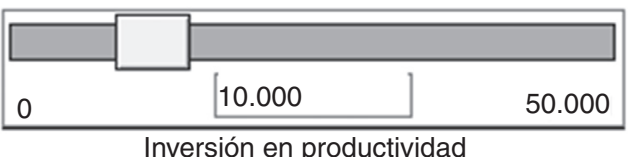

Inversión en productividad

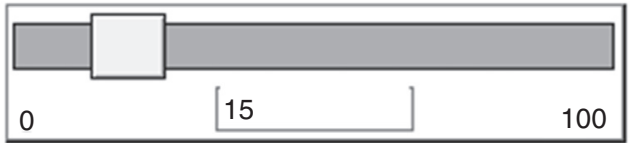

Empleados deseados

Figura 5. Tablero de control de entrada.

Tabla 1

Escenarios resultantes a partir de seis estrategias

\begin{tabular}{|c|c|c|c|c|c|c|}
\hline & 1. Base & $\begin{array}{l}\text { 2. Intensivo en } \\
\text { mano de obra }\end{array}$ & $\begin{array}{l}\text { 3. Intensivo en } \\
\text { productividad }\end{array}$ & $\begin{array}{l}\text { 4. Intensivo en } \\
\text { inventario }\end{array}$ & $\begin{array}{l}\text { 5. Intensivo en } \\
\text { capacidad }\end{array}$ & $\begin{array}{l}\text { 6. Intensivo en } \\
\text { publicidad }\end{array}$ \\
\hline $\begin{array}{l}\text { Inversión } \\
\text { publicidad }\end{array}$ & 10,000 & 10,000 & 10,000 & 10,000 & 10,000 & 30,000 \\
\hline $\begin{array}{r}\text { Capacidad } \\
\text { deseada }\end{array}$ & 100,000 & 100,000 & 100,000 & 100,000 & 150,000 & 100,000 \\
\hline $\begin{array}{c}\text { Cobertura deseada } \\
\text { de inventario }\end{array}$ & 2 & 2 & 2 & 6 & 2 & 2 \\
\hline $\begin{array}{l}\text { Inversión } \\
\text { productividad }\end{array}$ & 10,000 & 10,000 & 30,000 & 10,000 & 10,000 & 10,000 \\
\hline $\begin{array}{r}\text { Empleados } \\
\text { deseados }\end{array}$ & 15 & 50 & 15 & 15 & 15 & 15 \\
\hline VPN [M\$] & 30,140 & 21,808 & 20,874 & 30,391 & 27,233 & 30,626 \\
\hline Clientes & 3,670 & 3,684 & 3,670 & 3,670 & 3,670 & 4,326 \\
\hline
\end{tabular}

Las cinco variables decisionales, que reflejan la estrategia del emprendedor, se pueden modificar a través de un tablero de control de entrada (fig. 5), considerando las cuatro perspectivas del MB.

Se simularon seis escenarios, considerando valores extremos de las cinco variables decisionales, como se muestra en la tabla 1. El horizonte de simulación fue de 25 años. 

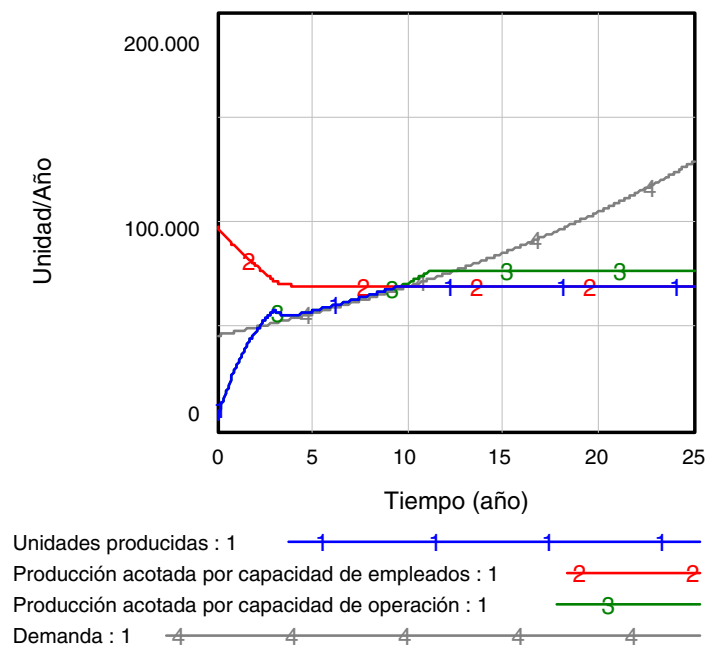

Figura 6. Producción y demanda, escenario base.
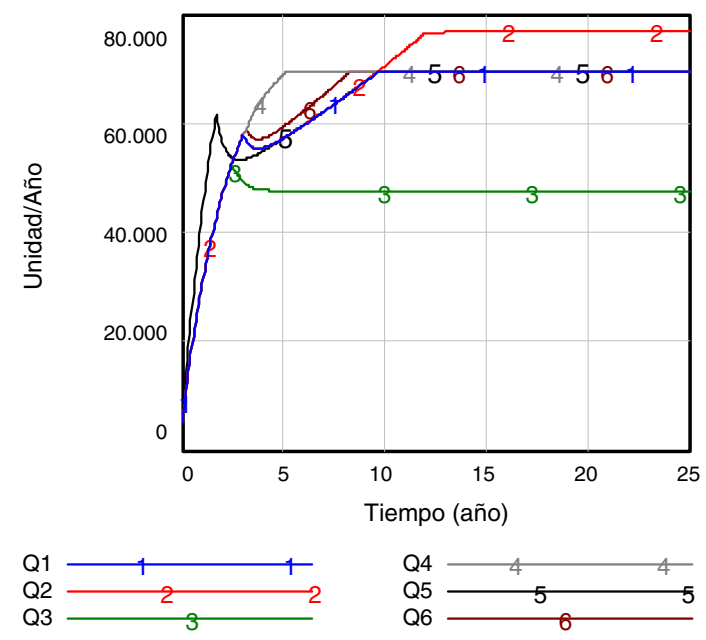

Figura 7. Producción por escenario.

La figura 6 muestra el comportamiento de la producción y la demanda para el escenario base. La producción se acota para todos los escenarios por las capacidades de producción o la de los empleados. La demanda se alcanza a satisfacer hasta el período 12.

La figura 7 muestra la producción para los seis escenarios considerados. En los primeros años existe un período de transición debido a los efectos combinados de los ajustes en capacidad, inventarios, mano de obra y productividad, definidos por sus correspondientes tiempos de corrección. La producción se trunca de forma diferente para cada uno de los escenarios debido al desarrollo desequilibrado de los diversos recursos considerados en el SVE.

El escenario intensivo en mano de obra es el que se trunca posteriormente debido a que al inicio existe capacidad sobrada en este rubro, lo que también explica que si se invierte en productividad o en empleados (escenarios 2 y 3 ) se desperdicia esta inversión y disminuye su correspondiente 


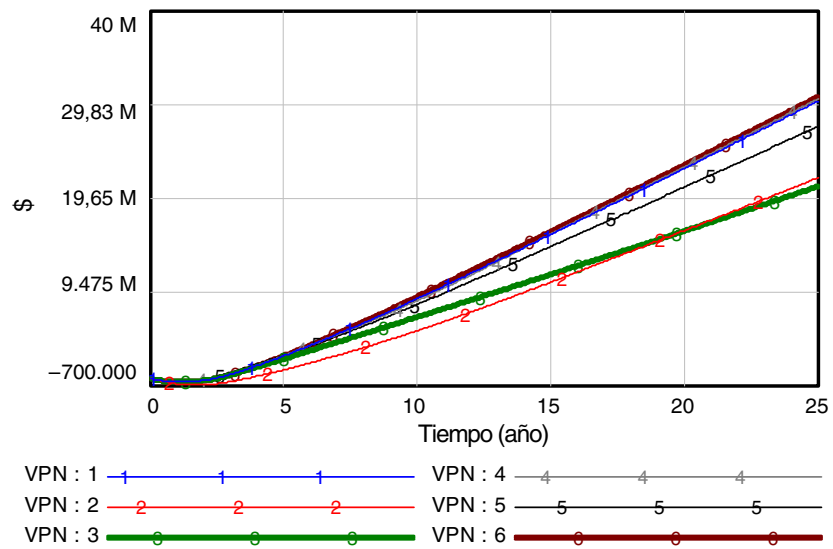

Figura 8. VPN por escenario.

VPN. En el escenario 5 no se trunca la producción. Estos acotamientos se deben al crecimiento desequilibrado entre los diversos recursos, producto de las estrategias definidas por el usuario del SVE. Si se acorta el tiempo de construcción de capacidad la restricción que trunca la producción se relaja, alcanzando a satisfacer de una manera más adecuada la demanda.

Como se muestra en la tabla 1 y figura 8, los menores VPN corresponden a los escenarios 3 y 2 , intensivos en productividad y mano de obra. El mayor VPN corresponde al escenario 6, intensivo en publicidad. En los primeros años los flujos son negativos para todos los escenarios, lo cual significa que es necesario contratar deuda para el financiamiento de las estrategias.

A medida que se avanza en la simulación los flujos van siendo positivos y crecientes, lo cual permite amortizar la deuda adquirida en períodos anteriores e ir incrementando utilidades retenidas producto de la operación y de los rendimientos obtenidos de sus correspondientes inversiones.

Al simular un séptimo escenario, considerando una estrategia agresiva al invertir en cada recurso las máximas cantidades mostradas en cada uno de los escenarios anteriores, se obtiene el VPN más bajo de 9,974 M\$, debido al exceso y desequilibrio en el desarrollo relativo de los recursos y los altos costos que esto implica. El VPN máximo de 33,829 M\$ se logra con la estrategia determinada por $[26,000 ; 104,000 ; 2.2 ; 13,000 ; 12]$, de acuerdo al orden de las variables decisionales del tablero de la figura 4. Este último VPN sería el objetivo a lograr de los participantes después de realizar su análisis de sensibilidad, simulando varias de sus estrategias propuestas.

Las figuras 6-8 permiten identificar que el desarrollo desequilibrado de los recursos considerados en las cuatro perspectivas del MB trunca o limita el total aprovechamiento de todos estos recursos. Estos cuellos de botella son el resultado de haber invertido recursos financieros de sobra en algunos recursos y han faltado en otros, llevando finalmente a la disminución del indicador de desempeño.

Al utilizar el SVE es posible identificar el impacto que tiene cada uno de los recursos en el VPN, mediante un análisis de sensibilidad. Cabe recordar que las relaciones causa-efecto de los recursos y la medida de desempeño son complejas y no lineales. La figura 9 muestra las gráficas de sensibilidad para cada una de las variables decisionales (desarrollo de recursos), considerando una variación de $\pm 20 \%$ en cada uno de ellos respecto a su correspondiente valor inicial. Este análisis de sensibilidad permite al usuario lograr un sentido de importancia relativa de cada uno de 


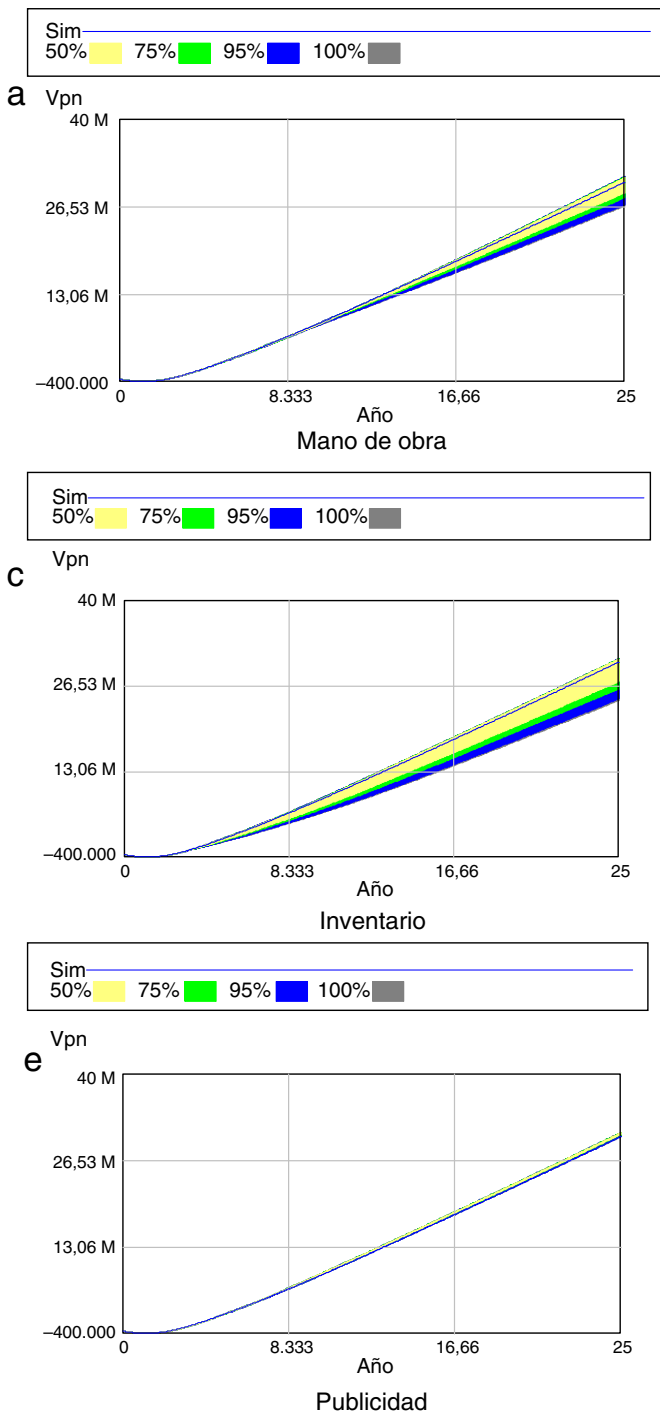

\begin{tabular}{|ll|l|}
\hline Sim & & \\
$50 \%$ & $75 \%$ & $95 \%$ \\
\hline
\end{tabular}
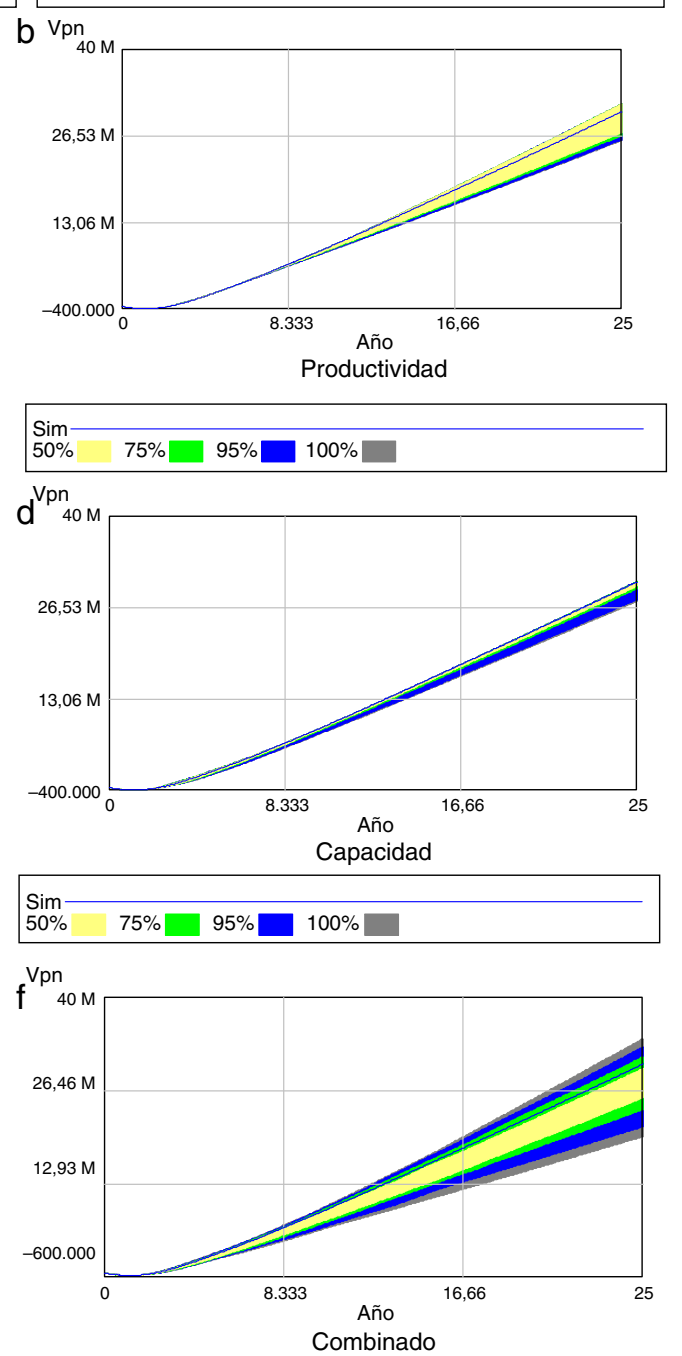

Figura 9. Análisis de sensibilidad.

9a. Mano de obra; 9b. Productividad; 9c. Inventario; 9d. Capacidad; 9e. Publicidad; 9f. Combinado

los recursos y puede guiar en sus decisiones de priorizar el desarrollo de ciertos recursos respecto a otros.

Como puede observarse en la figura 9e, el recurso menos sensible, es decir, el que tiene menor dispersión, es la inversión en publicidad. El recurso que muestra mayor sensibilidad es la cobertura de inventario (fig. 9c). La figura 9f muestra la sensibilidad de los cinco recursos combinados, la cual es mayor que la de cada uno considerado individualmente.

De acuerdo a Warren (2008, cap. 4), el desarrollo desequilibrado de recursos en una empresa con recursos múltiples conduce a una situación de crecimiento ineficiente, resultado de una deficiente planeación estratégica. Los niveles de los recursos deben construirse equilibradamente debido a 
que estos son interdependientes (ver la sección «El emprendedor y el manejo de recursos») en la empresa. El desarrollo de un recurso depende de las decisiones de gestión, de su propio nivel y de los niveles relativos de los otros recursos estratégicos de la empresa (Warren, 2008). La falta de capacidad de coordinación, integración y asignación de montos y tiempos de inversión, en el caso de desarrollo de empresas con recursos múltiples, (Grant, 2013, caps. 5 y 6), involucra aspectos intangibles, como la capacidad de aprendizaje de la empresa y la cultura organizacional, así como aspectos tangibles y más formales, como la estructura organizacional, la asignación de puestos y responsabilidades interfuncionales, el diseño de los sistemas administrativos, de incentivos y de información, y la evaluación y asignación de recursos financieros al desarrollo de los diversos recursos estratégicos. El desarrollo de recursos es una actividad estratégica que involucra una adecuada planeación estratégica, incluyendo explícitamente la determinación de la ventaja competitiva, así como la contribución de cada recurso al logro de esta, a la rentabilidad de la empresa y a las acciones de la competencia (Kunc y Morecroft, 2010).

\section{Conclusiones}

La creación de una nueva empresa representa un proceso dinámico, en el cual los emprendedores evalúan sus estrategias de acuerdo a los resultados que van teniendo conforme avanza su proyecto, tomando en cuenta que la mejor estrategia a implementar en su empresa va a depender de factores tanto internos - como el desarrollo de sus recursos - como externos - las características y tendencias de su entorno y mercado meta.

El SVE propuesto podría permitir al emprendedor sensibilizarse en el proceso de planeación estratégica, buscando el logro de una toma de decisiones más informada que la perspectiva tradicional, identificando y analizando los principales recursos con los que cuenta y sus relaciones causales con los objetivos estratégicos y sus indicadores de desempeño. Al enmarcar este análisis dentro de las cuatro perspectivas del MB que conforman el nuevo negocio (recursos humanos, procesos, mercado y finanzas), le permite al emprendedor conocer y discutir con otros interesados las relaciones existentes entre estos recursos y sus indicadores de desempeño, analizando el posible efecto de sus decisiones.

El SVE considera las acumulaciones de recursos en el tiempo y permite modificar y evaluar diversas estrategias conforme el estratega vaya comprendiendo su estructura y funcionamiento. El SVE propuesto permite conectar todas las áreas involucradas en el negocio, con la posibilidad de modificar el modelo mental del estratega desde una visión holística, sistémica y equilibrada, lo cual puede servir también como un método interactivo explícito de aprendizaje entre el emprendedor y otros participantes interesados en el proceso de planeación estratégica de su proyecto de empresa.

De acuerdo a los objetivos didácticos que se pretendan lograr utilizando el SVE, es posible agregar más variables decisionales en el SVE, como el tiempo de construcción de la nueva capacidad de producción, el tiempo de ajuste del inventario, el tiempo para surtir los pedidos y el tiempo de contratación y capacitación de nuevos empleados, entre otras. Añadir estas variables decisionales implicaría solamente ampliar el tablero de control de entrada en el SVE propuesto.

Con el objetivo de aplicar el SVE propuesto, es necesario diseñar un ambiente de aprendizaje adecuado al perfil de los participantes o usuarios, bajo el esquema de aprendizaje experiencial (Domenge, 2009). Se recomienda desarrollar un caso didáctico que coloque al participante como parte del equipo de estrategas de una empresa, con la información que requiera respecto a la industria en la que se encuentra. En dicho caso didáctico se deberán incluir información que permita discutir e identificar los recursos estratégicos de la empresa, así como las relaciones causales con los indicadores de desempeño (VPN), como se muestra en el diagrama de la figura 3. 
Finalmente, se recomienda incluir una serie de cuestionamientos estratégicos que conduzcan a los usuarios, mediante el uso del SVE, a identificar la importancia relativa del desarrollo de los recursos de la impresa respecto a su impacto en el VPN, así como el planteamiento de una estrategia concreta para su desarrollo.

\section{Agradecimientos}

Se agradece a la Asociación Mexicana de Cultura, A.C. por el apoyo brindado en la elaboración de la presente investigación.

\section{Anexo 1. Simuladores de vuelo ejecutivos}

\begin{tabular}{|c|c|c|c|c|c|c|}
\hline \multicolumn{7}{|c|}{ SIMULADORES PARA EMPRENDEDORES } \\
\hline MODELO & REFER ENCIA & OBJETIVO & M ÓDULOS & $\begin{array}{c}\text { VARIABLES } \\
\text { DECISIONALES }\end{array}$ & $\begin{array}{l}\text { MEDIDAS DE } \\
\text { DESEMPENNO }\end{array}$ & COMENTARIOS \\
\hline $\begin{array}{l}\text { A Systems Dynamics } \\
\text { Model of a Startup }\end{array}$ & Saad, 2013 & \begin{tabular}{|l|}
$\begin{array}{l}\text { Simular las dinámicas internas entre recursos } \\
\text { humanos, desarrollo del producto, clientes y } \\
\text { perspectiva financiera, y conceptual zar éstas } \\
\text { y las dinámicas externas relacionadas con la } \\
\text { creación de nuevos negocios }\end{array}$ \\
\end{tabular} & $\begin{array}{l}\text { Recursos Humanos, Producción, } \\
\text { Clientes, Finanzas }\end{array}$ & $\begin{array}{c}\text { Contratación, salario, } \\
\text { inversión en productos y } \\
\text { cambios en los mismos, } \\
\text { inversión en publicidad y } \\
\text { promociones, precio } \\
\end{array}$ & $\begin{array}{l}\text { Ventas, clientes, } \\
\text { crecimiento, ingresos }\end{array}$ & $\begin{array}{l}\text { Abarca las distintas áreas involucradas en la creación } \\
\text { de una nueva empresa y cómo las decisiones tomadas } \\
\text { impactan en el crecimiento de la misma. }\end{array}$ \\
\hline EcoRoll & Bianchi, Bivona \& Landriscina, 2000 & $\begin{array}{l}\text { Planeación de nuevos negocios en su etapa } \\
\text { inicial y de crecimiento. El objetivo es el } \\
\text { análsis de la producción y venta de patines. }\end{array}$ & Mercadotecnia, Producción, Finanzas & $\begin{array}{c}\text { Capacidad, Publicidad y } \\
\text { Promoción, Precio, } \\
\text { Nuevos productos } \\
\end{array}$ & \begin{tabular}{|c|} 
Estados Financieros, \\
Ingresos, Total Clientes, \\
Utilidades \\
\end{tabular} & $\begin{array}{l}\text { Se enfoca al desarrollo de un plan de negocios } \\
\text { especifico. Busca que haya un balance entre lo } \\
\text { comercial y la producción. }\end{array}$ \\
\hline $\begin{array}{l}\text { Threshold } \\
\text { Entrepreneur }\end{array}$ & hitp:l/lwww. prenhall.com/threshold/ & $\begin{array}{l}\text { Desarrollo de habilidades gerenciales para } \\
\text { start-ups }\end{array}$ & Mercadotecnia, Operaciones y Finanzas & \begin{tabular}{|l} 
Estimación de ventas, \\
precio, desarrollo de \\
tecnologia y uso de \\
estrategias de publicidad
\end{tabular} & $\begin{array}{c}\text { Ventas, participación de } \\
\text { mercado }\end{array}$ & $\begin{array}{l}\text { Los jugadores no tienen la flexibilidad de escoger la } \\
\text { industria. Además, no se enfoca a la planeación inicial, } \\
\text { sino en cómo administrar una nueva compañía. }\end{array}$ \\
\hline The Business Disc & $\frac{\text { hitp://online.sfsu.edu/ } / \text { cmgaglio/BizDiscSimul }}{\text { aton.PDF }}$ & \begin{tabular}{|l}
$\begin{array}{l}\text { Los jugadores deben desarrollar un plan de } \\
\text { negocios para una nueva companíia. Después } \\
\text { de ello, el programa simulará operaciones por } \\
1 \text { año. }\end{array}$ \\
\end{tabular} & $\begin{array}{l}\text { Producción, Recursos Humanos, } \\
\text { Finanzas, Mercadotecnia }\end{array}$ & $\begin{array}{l}\text { Tipo de negocio, } \\
\text { localización, } \\
\text { requerimientos de } \\
\text { inversión, seguros, } \\
\text { publicidad. }\end{array}$ & $\begin{array}{l}\text { Estados Financieros, } \\
\text { Proyección de gastos. }\end{array}$ & $\begin{array}{l}\text { Se enfoca mucho en el desarrollo del plan de } \\
\text { negocios, lo cual es muy positivo, pero la simulación } \\
\text { sólo aplica para } 1 \text { año de operaciones. }\end{array}$ \\
\hline Goventure & www.goventure.net & \begin{tabular}{|c|} 
Los jugadores actúan como emprendedores \\
que están estableciendo una nueva compañia \\
y deben manejarla existosamente para que \\
vaya de acuerdo a sus objetivos personales. \\
Los jugadores deben manejar sus negocios, \\
recibir consejos, atender seminarios de \\
entrenamiento y tomar decisiones sobre \\
cerrar o vender sus compañias.
\end{tabular} & $\begin{array}{l}\text { Producción e Inventario, Mercadotecnia, } \\
\text { Finanzzas, Recursos Humanos }\end{array}$ & $\begin{array}{c}\text { Precio, Inventario, } \\
\text { Inversión en Publicidad, } \\
\text { Contratación y motivación } \\
\text { de empleados }\end{array}$ & $\begin{array}{l}\text { Estados Financieros, Nivel } \\
\text { de efectivo, clientes }\end{array}$ & $\begin{array}{l}\text { El programa incluye la etapa de planeación, el } \\
\text { establecimiento de la companaía yel desarrollo de la } \\
\text { misma e involucra los objetivos personales de los } \\
\text { jugadores. Un programa completo, pero que se enfoca } \\
\text { mucho en la vida personal. }\end{array}$ \\
\hline VCommerce & $\frac{\text { http://misweb.bus.msu.edufacc890/resources }}{\frac{\text { Ivc-EntreGuide.pdf }}{2}}$ & $\begin{array}{l}\text { Un pequeño gruo de emprendedores diseñará } \\
\text { e implementará algunas compañías que usan a } \\
\text { red para vender bienes y servicios a } 200-300 \\
\text { usuarios. }\end{array}$ & Mercadotecnia, Legal, Finanzas & $\begin{array}{l}\text { Inversión en capital, } \\
\text { Estimación de ventas, } \\
\text { Investigación de } \\
\text { mercados. }\end{array}$ & $\begin{array}{l}\text { Comparación entre } \\
\text { estimaciones y resultados } \\
\text { actuales, flujo de efectivo. }\end{array}$ & 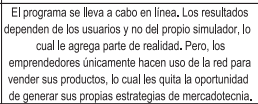 \\
\hline Venture & Venture.SIM & $\begin{array}{l}\text { Simulador disen̈ado para cubrir las } \\
\text { necesidades de incorporar los cursos de } \\
\text { finanzas emprendedoras y finanzzas } \\
\text { corporativas. } \\
\end{array}$ & Finanzas Corporatvas & $\begin{array}{l}\text { Valuación de activos } \\
\text { financieros, opciones. }\end{array}$ & $\begin{array}{l}\text { Flujos de efectivo, } \\
\text { Estadisticas. }\end{array}$ & $\begin{array}{l}\text { Se enfoca únicamente en las frnanzas de la companía, } \\
\text { por lo que su alcance es muy linitado. }\end{array}$ \\
\hline Entrepreneur & hitp:Thome. att.net-1-simulations/entre2.htmlas & $\begin{array}{l}\text { Involucra la operación de una tienda de ropa. } \\
\text { Los jugadores actúan como administradores } \\
\text { para la toma de decisiones que impactarán en } \\
\text { peraciones de la tienda. Los jugadores } \\
\text { escogen entre diferentes lineas de ropa, } \\
\text { establecen precios, los gastos de publicidad y } \\
\text { promocionesy cuánto comprar cada periodo. }\end{array}$ & $\begin{array}{l}\text { Finanzas, Mercadotecnia, Recursos } \\
\text { Humanos }\end{array}$ & $\begin{array}{l}\text { Compras, Linea de ropa, } \\
\text { precio, presupuesto } \\
\text { promocional y para } \\
\text { publicidad, salario, polfica } \\
\text { de devoluciones, } \\
\text { investigación de mercados, } \\
\text { dividendos, préstamos. }\end{array}$ & $\begin{array}{l}\text { Estados Financieros, } \\
\text { Precio de la acción }\end{array}$ & $\begin{array}{l}\text { Los jugadores no deciden qué tipo de negocio } \\
\text { establecer, pero resulta un buen programa para } \\
\text { negocios de venta de productos. }\end{array}$ \\
\hline DEAL & hittp://wwww.towson.edu/ precha/deal_1.htmser & $\begin{array}{l}\text { Los jugadores pueden establecer compañias } \\
\text { en } 5 \text { industrias interdependientes con el fin d } \\
\text { drectores, administradores, agentes de } \\
\text { compras, agentes de ventas y clientes de las } \\
\text { companías. }\end{array}$ & Ventas, Finanzas, Clientes & Inversión en capital & Estadisticas & $\begin{array}{l}\text { No hay mucha información dispnible para este } \\
\text { programa. El simulador le permite al usuario escoger } \\
\text { su posición en la compañia. }\end{array}$ \\
\hline Corporation & $\begin{array}{c}\text { http:/home.att.net/-simulations/corp/corpwe } \\
\text { b.htm }\end{array}$ & 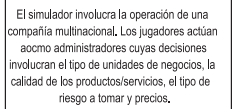 & $\begin{array}{l}\text { Finanzas, Recursos Humanos, } \\
\text { Mercadotecnia }\end{array}$ & \begin{tabular}{|c|} 
Préstamos, Precio, \\
Presupuestos, Expansión
\end{tabular} & Ventas & $\begin{array}{l}\text { El simulador se enfoca en el desarrollo de una gran } \\
\text { compañía en lugar de una nueva empresa, por lo que } \\
\text { no cubre el objetivo. }\end{array}$ \\
\hline
\end{tabular}

\section{Referencias}

Akkermans, H. y Oorschot, K. (2005). Relevance assumed: A case study of balanced scorecard development using system dynamics. Journal of the Operational Research Society, 56(8), 931-941.

Aleven, V., Stahl, E., Schworm, S., Fischer, F. y Wallace, R. (2003). Help seeking and help design in interactive learning environments. Review of Educational Research, 73, 277-320.

Barney, J. y Hesterly, W. (2012). Strategic management and competitive advantage: Concepts (4th edition). Upper Saddle River, NJ: Prentice Hall.

Bianchi, C. (2002). Introducing SD modelling into planning and control systems to manage SME's growth: A learningoriented perspective. System Dynamics Review, 18, 315-338. http://dx.doi.org/10.1002/sdr.258 
Bianchi, C., Bivona, E. y Landriscina, F. (2000). Promoting Entreprenership Thorugh Open-Distance-Learning Management Flight Simulators EcoRoll Educational Package.

Bianchi, C., Winch, G. y Grey, C. (1998). The Business Plan as a Learning-Oriented Tool for Small/Medium Enterprises: A Business Simulation Approach.

Blue Ocean Strategy Simulation, BOSS [consultado 8 Sep 2015]. Disponible en: http://web.stratxsimulations. $\mathrm{com} /$ simulation/business-strategy-simulation

Capelo, C. (2009). A system dynamics-based simulation experiment for testing mental model and performance effects of using the balanced scorecard. System Dynamics Review, 25, 1-34. http://dx.doi.org/10.1002/sdr.413

Carroll, G. R. (1993). A sociological view on why firms differ. Strategic Management Journal., 14, $237-249$.

Choi, J. y Hannafin, M. (1995). Situated cognition and learning environments: Roles, structures, and implications for design. Educational Technology Research and Development, 43, 53-69.

Davidsen, P. (2000). Issues in the design and use of system-dynamics-based interactive learning environments. Simulation Gaming, 31, 170-177. http://dx.doi.org/10.1177/104687810003100204

Dollinger, M. (2008). Entrepreneurship Strategies and Resources (4th edition). Illinois, IL: Marsh Publications.

Domenge, R. (2009). Importancia de los factores de transferencia de contenido en una escuela de negocios: percepciones de estudiantes y profesores. Cuadernos de Estudios Empresariales, 19, 75-104.

Domenge, R. y Belausteguigoitia, I. (2009). Nuevas PyMEs: problemas y recomendaciones. Dirección Estratégica, 29(8), 25-27.

Domenge, R. y Vidal, D. (2015). Mejora de la planeación estratégica mediante los simuladores de vuelo ejecutivos. Dirección Estratégica, 53.

GEM, Global Entrepreneurship Monitor. 2012. Monitor Global de la Actividad Emprendedora México 2012 [consultado 19 Feb 2014]. Disponible en: http://www.gemconsortium.org/docs/3076/gem-mexico-2012-report

Grant, R. (2013). Contemporary Strategy Analysis (8th edition). Wiley.

Gumbus, A. y Lussier, R. (2006). Entrepreneurs use a balanced scorecard to translate strategy into performance measures. Journal of Small Business Management, 44, 407-425.

Kaplan, R. y Norton, D. (1992). The balanced scorecard-measures that drive performance. Harvard Business Review, 70 , 71-79.

Kolb, D. (2014). Experiential learning: Experience as the source of learning and development (2nd edition). Pearson.

Kopainsky, B., Alessi, S., Pedercini, M. y Davidsen, P. (2009). Measuring knowledge acquisition in dynamic decision making tasks.

Kunc, M. H. y Morecroft, J. D. W. (2010). Managerial decision making and firm performance under resource-based paradigm. Strategic Management Journal, 31, 1164-1182. http://dx.doi.org/10.1002/smj.858

Lane, D. (1995). On a resurgence of management simulations and games. The Journal of the Operational Research Society, 46, 604-625.

Maier, F. H. y Gröbler, A. (2000). What are we talking about? — A taxonomy of computer simulations to support learning. Systems Dynamics Review, 16(2), 135-148.

Markstrat [consultado 8 Sep 2015]. Disponible en: http://web.stratxsimulations.com/simulation/strategic-marketingsimulation/

MIT Business Strategy Games [consultado 8 Sep 2015]. Disponible en: http://web.stratxsimulations.com/ simulation/overview/

Ogatta, K. (2009). Modern Control Engineering (5th edition). Prentice Hall.

Pina, M. (2007). Entrepreneurship as decision making: Rational, intuitive and improvisational approaches. Journal of Enterprising Culture, 15, 1-20.

Rieber, L. (1996). Seriously considering play: Designing interactive learning environments based on the blending of microworlds, simulations and games. Educational Technology Research and Development, 44, 43-58.

Ritchie-Dunham, J. (2001). Informing Mental Models for Strategic Decision Making with ERPs and the Balanced Scorecard: A Simulation-Based Experiment.

Rogers, E. M. (1962). Diffusion of Innovations. Glencoe: Free Press.

Rugman, A. M. y Verbeke, A. (2002). Edith Penrose's Contribution to the Resource-Based View of the Strategic Management. Strategic Management Journal, 23, 769-780. http://dx.doi.org/10.1002/smj.240

Saad, F. (2013). Modeling and Comparing a Startup Dynamics in the US and Egypt.

Senge, P. (1994). The Fifth Discipline: The Art and Practice of the Learning Organization. Doubleday.

Sterman, J. (2007). Getting big too fast: Strategic dynamics with increasing returns and bounded rationality. Management Science, 53(4), 683-696.

Sterman, J. (2000). Business Dynamics: Systems Thinking and Modeling for a Complex World. Boston, MA: McGraw-Hill. 
Timmons, J. (2009). New Venture Creation: Entrepreneurship for the 21 st Century (8ht edition). New York, NY: McGrawHill.

Ventana Systems. Vensim [consultado 8 Sep 2015]. Disponible en: http://vensim.com/

Warren, K. 1997. The professional service microworld [consultado 8 Sep 2015]. Disponible en: http://www.strategydynamics.com/microworlds/professional-services/

Warren, K. (2005). Improving strategic management with the fundamental principles of system dynamics. System Dynamics Review, 21(4), 329-350. http://dx.doi.org/10.1002/sdr.325

Warren, K. (2008). Strategic Management Dynamics. Chichester, England: John Wiley \& Sons.

Wawer, M. (2010). Business simulation games in forming of student's entrepreneurship. International Journal of EuroMediterranean Studies, 3, 49-71. 